\title{
Automatic detection of osteosarcoma based on integrated features and feature selection using binary arithmetic optimization algorithm
}

\author{
Priti Bansal, et al. [full author details at the end of the article]
}

Received: 20 May 2021 / Revised: 19 December 2021 / Accepted: 3 January 2022 Published online: 7 February 2022

(C) The Author(s), under exclusive licence to Springer Science+Business Media, LLC, part of Springer Nature 2022

\begin{abstract}
Osteosarcoma is one of the most common malignant bone tumors mostly found in children and teenagers. Manual detection of osteosarcoma requires expertise and it is a labour-intensive process. If detected on time, the mortality rate can be reduced. With the advent of new technologies, automatic detection systems are used to analyse and classify medical images, which reduces the dependency on experts and leads to faster processing. In this paper, an automatic detection system: Integrated Features-Feature Selection Model for Classification (IF-FSMC) to detect osteosarcoma from the high-resolution whole slide images (WSIs) is proposed. The novelty of the proposed approach is the use of integrated features obtained by fusion of features extracted using traditional handcrafted $(\mathrm{HC})$ feature extraction techniques and deep learning models (DLMs) namely EfficientNet-B0 and Xception. To further improve the performance of the proposed system, feature selection (FS) is performed. Here, two binary variants of recently proposed Arithmetic Optimization Algorithm (AOA) known as BAOA-S and BAOA$\mathrm{V}$ are proposed to perform FS. The selected features are given to a classifier that classifies the WSIs into Viable tumor (VT), Non-viable tumor (NVT) and nontumor (NT). Experiments are performed to compare the performance of proposed IF-FSM-C to the classifiers which use HC or deep learning features alone as well as state-of-the-art methods for osteosarcoma detection. The best overall accuracy of $96.08 \%$ is obtained when integrated features extracted using HC techniques and Xception are used. The overall accuracy is enhanced to $99.54 \%$ after applying BAOA-S for FS. Further, the application of BAOA-S for FS reduces the number of features with the best model having only 188 features compared to 2118 features if no FS is applied.
\end{abstract}

Keywords Osteosarcoma - Whole slide images · Binary arithmetic optimization algorithm • Integrated features $\cdot$ EfficientNet-B0 $\cdot$ Xception

Kshitiz Gehlot and Abhishek Singhal are co-authors who contributed equally to this research. 


\section{Introduction}

Osteogenic Sarcoma or Osteosarcoma is a form of bone cancer that typically starts growth in long bones of legs and arms [39]. However, it can grow in any bone in the bone cells and in rare cases can affect the soft tissues around the bones. It mainly affects people in their teens or young adults, but may infect other age groups as well. Osteosarcoma is the most usual form of malignant tumour occurring in youth and average age of diagnosis for the disease is 15 years. Till late adolescence i.e., 15-19 years, the boys and girls are prone to get infected almost equally and beyond 15 years of age boys are more generally affected and susceptible to be attacked in the bone cells. The cause of osteosarcoma is unknown and it typically do not run-in families, but much like many other forms of cancers it occurs due to a fault in the gene which can cause this disease. Also, it has been linked to cases wherein there is a family history of familial retinoblastoma, a childhood cancer of the eye [34]. Children with familial retinoblastoma have a high risk for osteosarcoma in adolescence. Classic symptoms of the disease include pain in the affected bone, with initially a lower degree of pain and becoming excruciating with time. One of the other symptoms is bone fracture, as the affected bone weakens due to infection and losing its strength.

The overall 5-year survival rate in case of osteosarcoma is $68 \%$ and $67 \%$ for children ages 0 to 14 years and teens ages 15 to 19 years respectively [51]. If osteosarcoma is detected and treated timely before it has spread outside the area where it started, the general 5-year survival rate for people of all ages is 74\% [41]. If it has spread outside of the bones and into surrounding tissues or organs and/or the regional lymph nodes, the 5-year survival rate decreases to $66 \%$ which further declines to $27 \%$ if it spreads to distant parts of the body [41]. Looking at the low survival rate if it is not diagnosed on time, it can be inferred that it is important to diagnose and treat this disease as quickly as possible as early detection improves the chances of survival.

The task of osteosarcoma detection requires a lot of expertise, and is tiresome and timeconsuming if done manually. With the advent of new technology and faster processing systems, medical field has switched to automated detection systems which have the capability to predict the possibility of cancer being benign or malignant with some certainty. These systems have a general architecture of feature extractor followed by a classifier which takes the features as input. Some of the systems may also include a pre-processing stage before the feature extractor, which is required to improve the quality of the images by cleaning the data being fed into the model. This pre-processing may have techniques such as: Noise Removal Filters, Contrast Enhancement, etc. All these techniques benefit in cases when the dataset is of poor quality and has blurred images as well. Sometimes, segmentation is also performed before feature extraction to remove the background information. Recently, a number of Computer-Aided Detection (CAD) systems [6, 17, 30, 31, 44, 61] have been developed by researchers to segment and diagnose osteosarcoma from medical images especially magnetic resonance imaging (MRI) and computed tomography (CT) scans. However, these systems are bounded by the limitations of MRI and CT scan.

The cancerous bone cells are more densely populated with their nuclei being close to each other as compared to normal cells and also, they are irregular in shape. The cancerous bone cells exhibit darker shades of blue and purple and differences are also observed in texture properties. This mandates the extraction of these microscopic features to improve the accuracy of osteosarcoma detection. However, the macroscopic information from MRI and CT scan do not provide sufficient differential analysis of the lesion. To overcome the limitations of MRI 
and CT scans, Whole Slide Images (WSIs) are being used by researchers nowadays to improve the accuracy of osteosarcoma detection. These cells are normally identified using Hematoxylin and Eosin (H\&E) staining of the tissue taken from the affected areas [25]. After staining, the specimens are put under a microscope for analysis by pathologists, by mounting them on glass slides. In the work presented in this paper, the dataset used has sufficiently high-quality Whole Slide Images (WSIs) [36], which are the digital form of conventional glass slides, and doesn't require any pre-processing. Here, WSIs are classified into Viable Tumor (VT), Non-Viable Tumor (NVT) and Non-Tumor (NT) as shown in Fig. 1.

Due to the presence of background and unwanted information such as: Muscles, Inkmarkings, folded or severed tissues or blurred regions, it becomes of utmost need to remove such information before the data is fed into the feature extractor. For this task, segmentation is used which can assign labels to each pixel based on the properties (color intensity, texture or position) and each label represents a different region. The correlation between different regions is minimum. This helps us to differentiate between foreground and background attributes in the image and remove the unwanted information.

There are large number of segmentation methods used in literature each having a different basis for segmenting the regions. Some basic techniques are: edge-based [58], clustering-based [47], thresholding techniques [46], watershed [14] and region-based [3]. Edge-based segmentation involves the application of an edge filter, followed by categorization of the pixels to edge and non-edge by the use of differential operators - Prewitt Operator, Sobel Operator etc. Clustering-based techniques such as K-means is a famous algorithm used for the purpose of segmentation that clusters the image into K clusters, with each cluster having similar characteristics and attributes. Thresholding such as Otsu thresholding, converts the image into discrete levels with each level indicating a different class of pixels. Watershed is another category of segmentation which makes use of topological interpretation of image, with the intensity levels of pixels treated as topology or elevation. These algorithms start by flooding the basins or areas having minima, and continue this process till each basin is full, and finally each basin is a separate region. It is quite similar to flood fill algorithms. Finally, region-based segmentation techniques can be seed-based and non-seed based. This categorization is done on the basis of the initial points taken as regions to start the process of segmentation.

The next step in automatic detection systems is feature extraction which can be handcrafted (HC) or deep learning (DL) features. Feature extraction is the process of reducing the dimensionality of the input data to represent the data in a more interpretable format which can be easily analysed [20,50]. These techniques are typically used for extraction of HC features which are specific properties of the image decided manually based on the attributes of

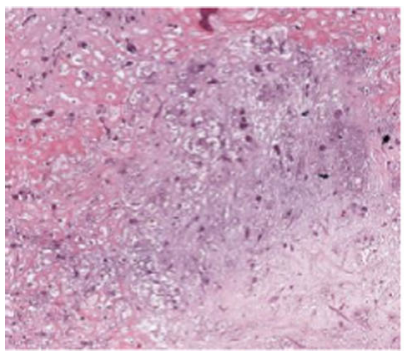

(a) Viable Tumor (VT)

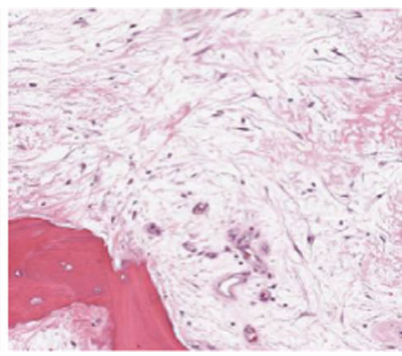

(b) Non-Viable Tumor (NVT)

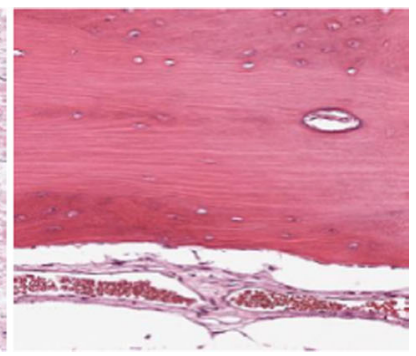

(c) Non-Tumor (NT)

Fig. 1 Sample Images from the Dataset. (a) Viable Tumor (VT). (b) Non-Viable Tumor (NVT). (c) Non-Tumor (NT) 
the target space. HC features have been widely used by researchers due to their simplicity of extraction especially in those cases where the data sets are small. These features can be decided by consultation from experts in the field under consideration. HC features do not require any form of training set for their derivation and can be more easily visualized as compared to other features. At the same time, with the use of HC features, different weights can be assigned to each feature based on the role they play in carrying out the targeted task.

However, in case of complex images these features become difficult to be decided upon and thus another form of feature extraction technique is employed namely deep learning models (DLMs) as feature extractors. DLMs have gained much importance in the last decade due to the recent developments seen in the field of computational power with the introduction of faster and compact computers, enabling the professionals to train deeper networks with ease and in lesser time. Typically, in this case Convolutional Neural Networks (CNNs) are employed as the DLM for the task of feature extraction. These models have the ability to automatically learn the features from the input image, but require a large training set with high variation for sufficient quality of attribute derivation. They have the ability to obtain higher number of low-level features which can describe the image in greater detail, thus helping in further analysis. DLM provides the benefit of no significant pre-processing requirement as they have the ability to carry out the same task automatically. But the downside of such models are their high computational and data requirements. Thus, it can be seen that the features extracted from both techniques HC and DLM have their pros and cons. Therefore, to take the advantage of the strength of both the methods, IF-FSM-C model is proposed that uses integrated features obtained by fusion of $\mathrm{HC}$ and DL features for classification.

Feature extraction is an essential step in any image processing step as the performance of a classifier is highly dependent on the features. However, not all features extracted from $\mathrm{HC}$ and DLM are important and contribute equally towards the classification process. The presence of irrelevant and redundant features may unnecessarily degrade the performance of the classifier. Hence, it creates the need for feature selection (FS) which involves further reducing the dimensionality of the solution space by taking the features which may conversely affect the decision-making ability of the model. This problem of selecting an optimal set of features can be converted into an optimization problem where the objective function is to minimize the loss on the training dataset. Formally, it can be framed as: Find an optimal subset of features from the entire set of features which can minimize the loss on the training dataset and in turn maximize the testing accuracy.

One of the main downsides of feature selection methods is that it requires a lot of time to try out each possible combination. Typically, if there are $\mathrm{n}$ features, then the number of possible solutions is: $\left(2^{n}-1\right)$. The number of solutions increase exponentially in powers of 2 . Therefore, brute force methods of FS will require a lot of time and computational power in order to reach a sufficiently optimal solution. Metaheuristic algorithms are a class of algorithms which have the ability to explore the solution space in smaller time and give nearly optimal solution in much lesser time than brute force approaches. Recently, metaheuristic algorithms have been applied by researchers in many applications and obtained optimal solutions to the real-world problems which were difficult to solve or required a lot of processing time using conventional algorithms. They have the ability to come out of locally optimal solutions due to the randomness in the algorithm. This motivates us to use metaheuristic algorithm for FS. Here, Arithmetic Optimization Algorithm (AOA) [2], a recently proposed metaheuristic algorithm, is used for the task of FS. AOA has been proved to be superior as compared to other metaheuristic algorithms in benchmark tests but it is aimed 
for continuous-valued problems. To tap into the strength of AOA, here a binary variant of the original AOA known as Binary Arithmetic Optimization Algorithm (BAOA) is proposed, so that it can be applied in binary optimization problems like FS problem. Two transfer functions namely S-shaped [32] and V-shaped [49] are used to make the continuous form of AAO applicable on binary search space. The respective binary variants are known as BAOA-S and BAOA-V. The main contributions of this paper are as follows:

1. An IF-FSM-C is proposed that uses integrated features obtained by the fusion of HC features and DLM features followed by FS to classify WSIs of osteosarcoma. Two deep learning models namely EfficientNet-B0 and Xception are trained as feature extractors and their performance is compared to each other in classifying WSIs.

2. Two binary variants of AAO known as BAOA-S and BAOA-V are proposed to solve feature selection problem.

The paper is organized as follows. Section 2 gives a brief overview of the related work. Section 3 gives a brief description of DLMs used in this paper. In Section 4, AOA is explained in brief. In Section 5, the proposed binary variants of AOA namely BAOA-S and BAOA-V are explained. The proposed approach IF-FSM-C to classify WSIs of osteosarcoma is presented in Section 6. In Section 7, experimental results are presented and discussed to analyse the performance of the proposed approach. In Section 8, conclusion and future work are discussed.

\section{Related work}

With the advancement in technology, automatic detection systems have been proposed by researchers for analysing and classifying medical images (X-ray, ultrasound images, MRI, CT scan, histological images etc.) to detect various kind of cancers and tumors. Some automatic detection systems $[11,33,48,53]$ classify medical images based on the features extracted using traditional handcrafted techniques whereas, others [13, 15, 16, 38, 54] use DLMs to perform feature extraction and classification of medical images. Recently, it has been observed that researchers have proposed automatic detection systems $[5,26,52,60]$ that use an amalgamation of both the techniques and these systems exhibit good performance as compared to their counter-parts. To further improve the performance of these systems FS is applied to the extracted features to remove irrelevant and redundant features thereby decreasing the number of features. A number of metaheuristic algorithms [1, 4, 8, 12, 21-23, 29, 40, 57] have been proposed by researchers in literature to perform FS. El-Kenawy [23] used Guided Whale Optimization Algorithm (Guided WOA) based on Stochastic Fractal Search (SFS) (SFSGuided WOA) for FS and applied on features extracted from chest CT images to diagnose COVID-19. Elgamal [21] proposed chaotic Harris Hawks Optimization (CHHO) and used for FS on 14 medical benchmark problems. Thaher [57] proposed Binary Harris Hawk Optimizer (BHHO) to perform FS on high dimensional dataset with low sample size. The results of $\mathrm{BHHO}$ have been validated on several datasets. A number of variants of Grey Wolf Optimizer (GWO) exist in literature for FS [1, 4, 22, 29]. As No-Free-Lunch (NFL) theorem [59] states that no optimization technique exists to solve all optimization problems which means that a metaheuristic algorithm may work well for some dataset but it may underperform for some other dataset, this motivated us to propose binary variant of recently proposed AOA for FS. 
Many automatic detection systems for osteosarcoma detection are proposed by researchers but as compared to other cancer detection systems, the literature on osteosarcoma is relatively less. Different segmentation methods $[6,17,31,44,61]$ are proposed for osteosarcoma segmentation in MRI and CT scans. Nasor and Obaid [44], proposed a technique to segment osteosarcoma in MRI images using a combination of image processing techniques which include K-means clustering, Chan-Vese segmentation, iterative Gaussian filtering, and Canny edge detection. Altameem [6] detected bone cancers using X-rays by applying the intuitionistic fuzzy rank correlation-based segmentation. After segmentation, different statistical features are extracted and are processed by applying the deep neural networks layers that successfully examines each features using Levenberg-Marquardt learning algorithm. The limitation of MRI and $\mathrm{CT}$ scan in capturing cellular information hinder the performance of CAD systems in detecting osteosarcoma. Unlike MRI and CT scans, WSIs provide better cell differentiation and microscopic information such as nuclei density, shape etc. can be easily extracted from them.

The dataset for osteosarcoma detection was proposed by Leavey et al. [36], consisting of digital WSIs. Arunachalam et al. [10], proposed a method to detect osteosarcoma by performing segmentation and handcrafted feature extraction on digital WSIs of osteosarcoma dataset. Mishra et al. [43] propose a CNN architecture to classify the dataset images into VT, NVT and NT. Furthermore, Mishra et al. [42] improved the CNN architecture by fine-tuning and augmenting the CNN to classify osteosarcoma WSIs dataset and then compared the results with AlexNet, VGGNet and LeNet. The image size was reduced to $128 \times 128$ patches, by cropping the original $1024 \times 1024$ image due to training limitations. An overall accuracy of 92.4\% is obtained which outperformed AlexNet, VGGNet and LeNet. Arunachalam et al. [9] combined machine learning and deep learning models to classify osteosarcoma WSIs. In the machine learning model, the segmentation was performed using K-means clustering followed by Otsu's multi-level thresholding to extract region of interests (ROIs) from the histological WSIs. Flood-fill algorithm is used to cluster the pixels and data analysis is performed to classify the WSIs into VT, NVT and NT. The segmented images are classified using a total of 13 machine learning models and the best accuracy was obtained on support vector machine (SVM). In deep learning, they built a custom CNN model based on AlexNet [35] and LeNet [37]. The number of samples in the dataset are increased using various data augmentation techniques such as rotation, flipping etc. Two sets of experiments are performed, one with patch size of $128 \times 128$ and other with tiles of size $1024 \times 1024$. Overall accuracy achieved on SVM was $89.9 \%$ whereas in deep learning models, patches yielded $93.3 \%$ and tiles yielded 91.2\%. Anisuzzaman et al. [7] proposed a deep learning framework based on VGG19 and InceptionV3, trained using transfer learning. The input fed to these models were WSIs with no patches, in order to improve the classification accuracy. However, they images have to be resized to $375 \times 375$ due to memory limitation. The overall accuracy was $93.91 \%$ and $78.26 \%$ on VGG19 and InceptionV3 models respectively on multiclass classification.

\section{Deep learning models}

Convolution Neural Networks (CNNs) are generally used for automatic information extraction from image data. They comprise of convolution layers that extract neighbourhood relationships, and encode them to form the outputs of neurons. Generally, denser CNNs are able to learn higher amount of information. But increasing density introduces some problems like 
vanishing gradient. Thus, many $\mathrm{CNN}$ architectures are proposed that handle such problems in unique ways, and hence can be used according to the requirement. Here, two architectures namely EfficientNet-B0 [56] and Xception [18] are used.

\subsection{EfficientNet-BO}

EfficientNet architectures are based on the idea of balanced scaling, to achieve the best possible results in available resources [56]. While most architectures were developed at a fixed budget, and later scaled with more available resources, EfficientNet architectures are designed by uniform scaling in depth, width and resolution to provide an optimized architecture for given resources. Thus, they are a progressive set of architectures, with increasing complexity. In simplest architecture EfficientNet-B0, the feature extraction model is made up of a convolution layer followed by 16 MobileNet [28] inverted bottleneck MBConv layers of size $3 \times 3$ or $5 \times 5$, followed by convolution, pooling and fully connected layers. It has least number of trainable parameters (5.3 million), which makes training easier and efficient in EfficientNet-B0.

\subsection{Xception}

Xception architecture [18] is inspired from ideology of Inception [55] model, that says, cross channel correlation and the spatial correlation of $\mathrm{N}$-dimensional data can be sufficiently decoupled so that they are not mapped jointly. Xception further improves it by completely decoupling cross channel and the spatial correlations. This is achieved using separable convolution layer. It also used shortcut connections between convolution layers, as proposed in ResNet [27] for identity mapping. The model consists of blocks and each block consists of at least two separable convolution layers of kernel size $3 \times 3$. The image size is reduced in initial blocks using MaxPooling of kernel $3 \times 3$, with convolution of kernel $3 \times 3$ with stride two in shortcut connections. The resultant model proved to be highly efficient for learning complex datasets.

\section{Arithmetic optimization algorithm}

Arithmetic Optimization Algorithm (AOA) is a metaheuristic algorithm recently proposed by Abualigah et al. [2]. It is based on the basic arithmetic operators, i.e., addition, subtraction, multiplication and division. Using these operators on a set of solutions, it can give the best element through mathematical optimization. The algorithm performs exploration using multiplication and division, as these operators can introduce change in large order. But these operators are unfit for local search due to high dispersion, so addition and subtraction operators are used to perform exploitation or local search.

AOA is a population-based algorithm, where initial solutions represented as $x_{i}=$ $\left[x_{i}^{1}, x_{i}^{2}, x_{i}^{d}\right]$, are generated randomly over a d-dimensional search space using Eq. 1.

$$
x_{i}^{j}=x_{\text {min }}^{j}+r\left(x_{\text {max }}^{j}-x_{\text {min }}^{j}\right)=\{1,2, ., N\}, j=\{1,2, ., d\}
$$

Where, $\mathrm{N}$ is the population size, $x_{i}$ represents the $i^{t h}$ solution, $x_{i}^{j}$ represents the $j^{\text {th }}$ dimension of the $i^{\text {th }}$ solution, $x_{\text {max }}^{j}$ and $x_{\text {min }}^{j}$ are the upper and lower bound in the search space for $j^{t h}$ dimension 
and $\mathrm{r}$ is a random number in the range 0 to 1 . The initial solution $X$ can also be represented by matrix as shown in Eq. (2).

$$
X=\left[\begin{array}{ccc}
x_{1}^{1} & \cdots & x_{1}^{\mathrm{d}} \\
\vdots & \ddots & \vdots \\
x_{N}^{1} & \cdots & x_{N}^{d}
\end{array}\right]
$$

A fitness function is defined which determines the quality of each solution in the population in an iteration. Here, the candidate solution with the highest fitness value in each iteration is considered as the most-optimal solution found so far. The decision regarding the selection of exploration and exploitation is based on Math Optimizer Accelerated (MOA) function calculated as shown in Eq. (3). It gives a coefficient based on current iteration $C_{\text {Iter }}$, that is used in the search phases.

$$
\operatorname{MOA}\left(C_{\text {Iter }}\right)=\operatorname{Min}+C_{\text {Iter }} \times\left(\frac{\text { Max }- \text { Min }}{M_{\text {Iter }}}\right)
$$

Where, $C_{\text {Iter }}$ denotes the current iteration, $M_{\text {Iter }}$ denotes the maximum number of iterations, Maxand Min are constants, denoting the maximum and minimum possible values of MOA respectively. MOA is designed in such a way so as to favour exploration in the initial stages and exploitation in the later iterations. A random number $r_{1} \in[0,1]$ is generated and its value is compared with MOA. If $r_{1}>M O A$, exploration is performed else exploitation.

\subsection{Exploration Phase}

During this phase, the solution space is explored using division and multiplication operators. For exploration, one of the division or multiplication operator is chosen randomly with equal probabilities. The new solution is calculated as shown in Eq. 4.

$$
x_{i}^{j}\left(C_{\text {Iter }}+1\right)=\left\{B\left(x^{j}\right) \div(M O P+\epsilon) \times\left(\left(x_{\max }^{j}-x_{\text {min }}^{j}\right) \times \mu+x_{\text {min }}^{j}\right), r_{2}\right.
$$

Where, $x_{i}^{j}\left(C_{\text {Iter }}+1\right)$ represents the $j^{\text {th }}$ dimension of the $i^{\text {th }}$ solution in the next iteration, $B\left(x^{j}\right)$ represents the $j^{\text {th }}$ dimension in the current best solution, $\epsilon$ is a small non-zero number, $\mu$ is a control parameter to adjust the search process and its value is set to 0.5 , and $r_{2}$ is a random number between $[0,1]$ and MOP is a Math Optimizer Function which is calculated in each iteration using Eq. 5.

$$
\operatorname{MOP}\left(C_{\text {Iter }}\right)=1-\frac{C_{\text {Iter }}^{1 / \alpha}}{M_{\text {Iter }}^{1 / \alpha}}
$$

Where, $\alpha$ is the sensitivity parameter whose value is set to 5 .

\subsection{Exploitation phase}

This phase represents the deep search of solution, i.e., the search of optimal solution near the best solution. Thus, the operators used are addition and subtraction operators. Similar to the 
exploration, the probability of selection of operators in exploitation is also equal. The new solutions are calculated as shown in Eq. 6.

$$
x_{i}^{j}\left(C_{\text {Iter }}+1\right)=\left\{B\left(x^{j}\right)-M O P \times\left(\left(x_{\text {max }}^{j}-x_{\text {min }}^{j}\right) \times \mu+x_{\text {min }}^{j}\right), r_{3}\right.
$$

Where, $r_{3}$ is a random number between $[0,1]$.

For further details on AOA, readers can refer [2].

\section{Binary Arithmetic Optimization Algorithm (BAOA-S/BAOA-V)}

In this section, the proposed binary variants BAOA-S and BAOA-V of AOA are presented. As mentioned by Kennedy and Eberhart [32], transfer function (TF) is an effective way of converting a continuous version of an algorithm into binary. The TF is used to convert given real value vector to binary vector. It defines the probability of changing the element in a solution vector to 0 or 1 based on the value of step vector. Here, two TFs: S-shaped (sigmoid) and V-shaped (hyperbolic tangent) are applied as shown in Fig. 2 to transform AOA into BAOA-S and BAOA-V respectively using Eqs. 7 and 8.

$$
\begin{gathered}
T_{S}\left(x_{i}^{j}\right)=\frac{1}{1+e^{x_{i}^{j}}} \\
\operatorname{TF}_{V}\left(x_{i}^{j}\right)=\left(\left|\tanh \left(\mathrm{x}_{\mathrm{i}}^{\mathrm{j}}\right)\right|\right)
\end{gathered}
$$

Where, $x_{i}^{j}$ represents the $j^{t h}$ dimension of the $i^{\text {th }}$ solution. In BAOA-S/BAOA-V, the position of the $i^{\text {th }}$ solution is updated using Eqs. 4 and 6 depending on the values of MOA, $r_{2}$ and $r_{3}$. The generated solution act as a step function and consists of real values. To convert this into a binary vector, either $T F_{S}$ or $T F_{V}$ is applied on the generated solution. Now, a random number $\mathrm{r}$ belonging to standard uniform distribution is generated and based on the value of the $T F$ and random number $r$, the real values in the solution vectors are mapped to either 0 or 1 as shown in Eqs. 9 and 10 for S-shaped and V-shaped TFrespectively.

$$
\begin{aligned}
& x_{i}^{j, b}\left(C_{\text {Iter }}+1\right)=\{1, r \\
& x_{i}^{j, b}\left(C_{\text {Iter }}+1\right)=\left\{x_{i}^{j}, r\right.
\end{aligned}
$$

Where, $x_{i}^{j, b}\left(C_{\text {Iter }}+1\right)$ is the binary vector obtained by mapping the step or real value solution vector $x_{i}^{j}\left(C_{\text {Iter }}+1\right)$ using $T F$.

\section{The proposed model: IF-FSM-C}

The proposed model is shown in Fig. 3 and the various steps are discussed in the following sections. 


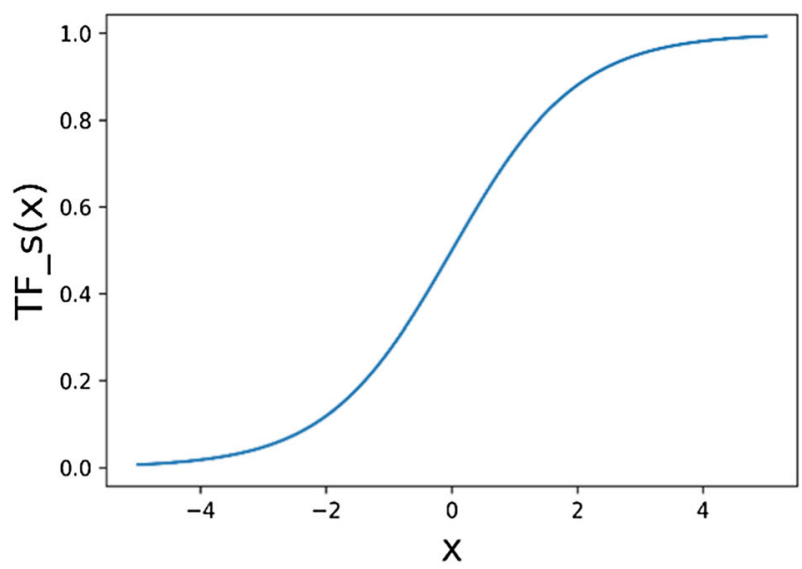

(a)

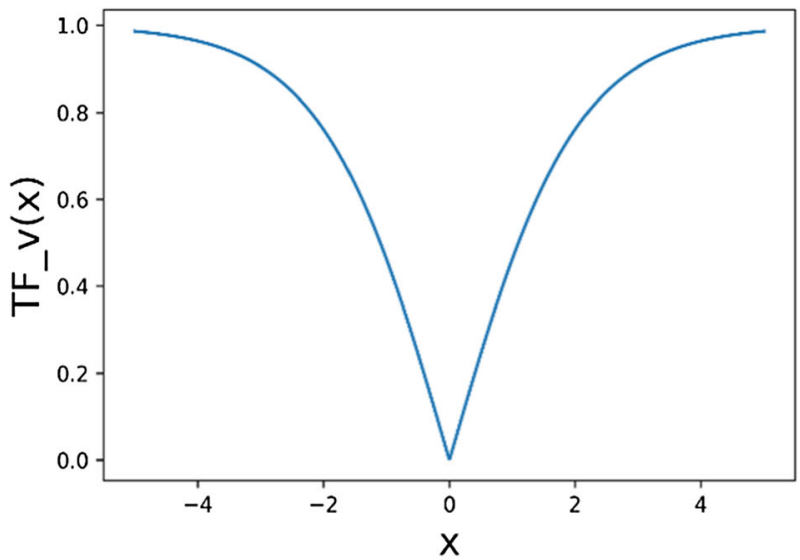

(b)

Fig. 2 Transfer Function (a) S-shaped and (b)V-shaped

\subsection{Image segmentation}

The original WSIs contain lot of information out of which some of the information is not important and do not contribute to the classification of the histological images. It is also possible that they may hamper the prediction of the classifier and is thus termed as "background or useless elements". In order to remove this form of information from the images, segmentation as a pre-processing stage is applied. The output of segmentation is the segmented image with all the unwanted information removed. The various forms of background information to be removed are: muscles, ink-markings, folded or severed tissues or blurred regions. The approach used in this paper for the segmentation is similar to that proposed by Arunachalam et al. [10]. Hue normalization is applied to the image by converting the RGB image into HSV, followed by multi-level thresholding in the hue channel. This image is then 


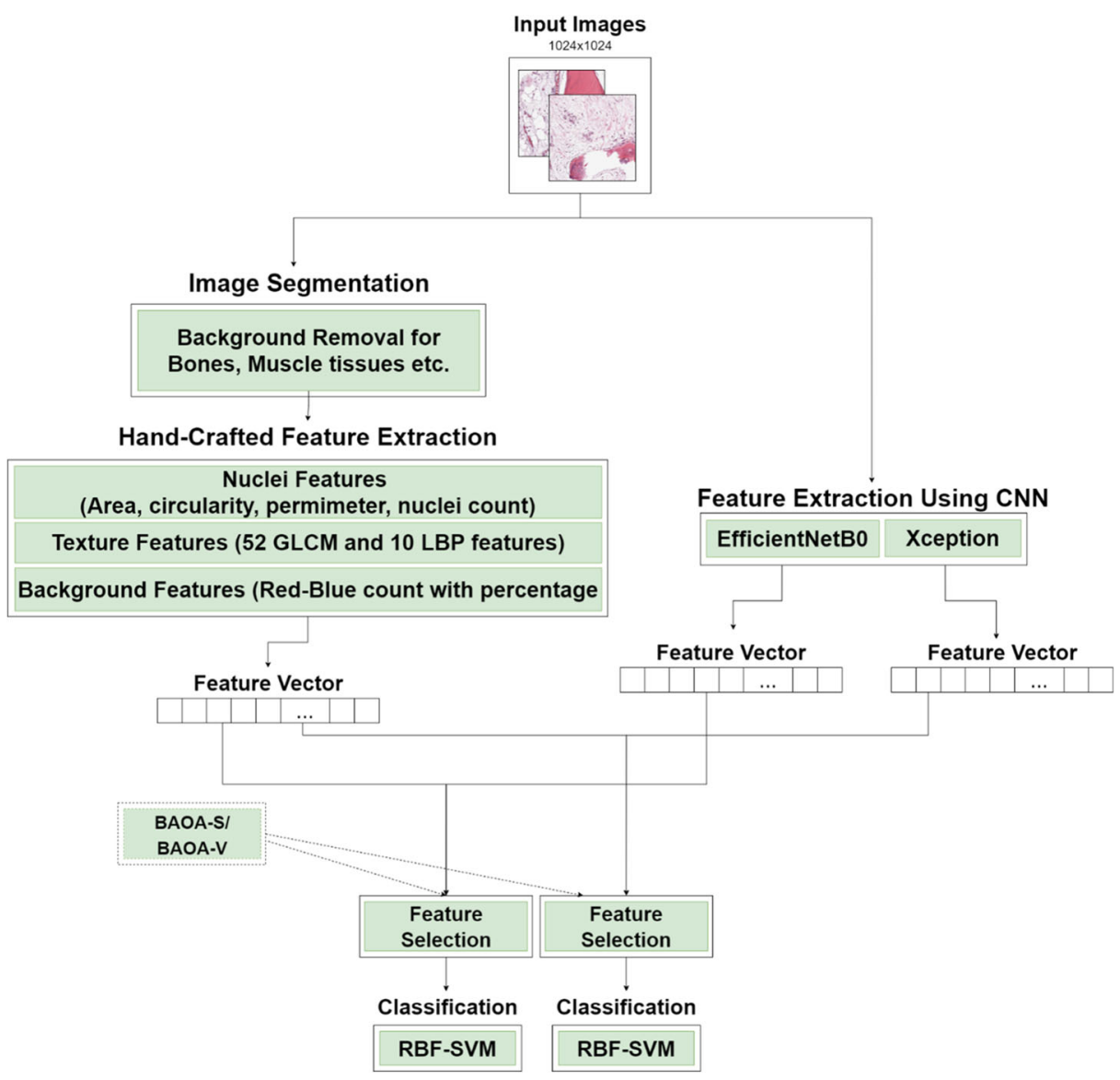

Fig. 3 The Proposed IF-FSM-C

converted back into the RGB form. The RGB image is subjected to K-means clustering segmentation for $\mathrm{K}=3$ (pink, blue and white clusters). The pink and blue clusters are normal and are required for further feature extraction, while the white cluster is the background information and needs to be removed from the image. For the removal of the white cluster, the pixels closest to the white centre are identified and are converted to white colour. The final output of this step is a modified image which has all the background information removed from it. Segmentation is important when HC feature extraction techniques are used for feature extraction. In case of DLM, segmentation is not mandatory as DLMs have the ability to segment and identify the region of interest in the image automatically.

\subsection{Feature extraction}

The performance of any classifier depends on the quality of features supplied to it. Thus, it is of utmost importance that sufficient and high-quality features are extracted from the image which are the closest representative of the attributes of the original image. Generally, the features extracted from the images are HC but with the advancement in the DLM, the use of $\mathrm{CNNs}$ has gained much importance showing significant improvements over the manual $\mathrm{HC}$ 
extraction. However, the use of HC features is still considered essential due to the freedom to select the attributes based on the properties of the image which the experts can observe and does not need any form of training for extraction process. In this research, an integrated approach where features are extracted using both HC techniques and DLMs is proposed.

\subsubsection{Handcrafted feature extraction}

Arunachalam et al. [9] proposed the use of various feature extraction techniques to obtain HC features such as: Nuclei Count, along with their shape and size, textural features using Gray-level co-occurrence matrix (GLCM) and red-blue pixel count and percentage. In this paper, local textural features of WSIs are proposed to be extracted using rotation-invariant Local Binary Patterns (LBP) [45]. Rotation-Invariant LBP allows us to analyse the local textural properties of an image. This is considered to be significant with respect to the typical characteristics of texture in images belonging to different categories. In case of VT, research have shown a higher density of nuclei, which can be detected by a higher value of the LBP operator. On the other hand, the density of the nuclei in cases of NVT and NT are lower with former having a relatively higher density. In the segmented image, the local textures are negligible in the white regions obtained by removing bones in the original image and all LBP values for the pixels would be similar and have slight to no variation at all. Thus, the use of textural properties derived from rotation-invariant LBP allows us to distinguish the images on the core feature of density of nuclei. A total of 10 features are extracted using $(8,1)$ neighbourhood. Global texture features are extracted using GLCM for four different orientations $0^{\circ}, 45^{\circ}, 90^{\circ}$ and $135^{\circ}$, each giving a total of 13 features. Thus, the number of global texture features are 52 .

Red-blue pixel count feature is used to represent the number of red and blue pixels in the image. In case of VT, the number of red and blue pixels are very high with a significant difference in their numbers. Here, there are more darker regions and less lighter regions. In case of NT, the pixels count is low and the difference between the count of red and blue pixels is also less. On the other hand, the pixel count is moderate in NVT but the difference between the counts is significant. Unlike VT, the high difference here signifies the presence of a greater number of lighter regions and less darker regions.

Finally, the nuclei characteristics such as: Nuclei Count, shape and size are extracted. The shape and size are measured using Area, Perimeter and Circularity of the different nuclei as shown in Eqs. $11-14$. In order to extract these features, it is imperative to identify and isolate the nuclei from the segmented images. For nuclei identification, region growing algorithm like watershed/flood fill is used, which marks the nuclei contours. The marked nuclei are used to extract the four features mentioned above.

$$
\text { Perimeter }=\sum_{i, j=1}^{X, Y} \text { boundary }(i, j)
$$

Where, $\mathrm{X}$ and $\mathrm{Y}$ are dimensions of the image and $\operatorname{boundary}(i, j)$ is calculated as:

$$
\operatorname{boundary}(i, j)=\left\{\begin{array}{c}
1, \text { ifConnectivityof } P_{i, j} \geq 2 \\
0, \text { otherwise }
\end{array}\right.
$$




$$
\text { Area }=\sum_{n_{i}=1}^{N} \text { Numberofpixelsinn }_{i}
$$

Where, $P_{i, j}$ is the pixel at position $(i, j), \mathrm{N}$ is the number of nuclei regions and connectivity represents the number of regions to which a given pixel $P_{i, j}$ is connected using 8-way connectivity.

$$
\text { Circularity }=4 \pi * \frac{\text { Area }}{\text { Perimeter }}
$$

A total of 70 features are extracted for each WSI using HC methods.

\subsubsection{DLMs as feature extractor}

The use of DLMs for the purpose of classification has been carried out numerous times and has also given significant results and improvements over the conventional models. The basic principle of the DLM is to break down the image into low-level features in initial layers and then combine those features to determine complex and high-level properties of an image. The classification from these features is carried out by using a dense layer to make predictions. The ability of the DLMs to learn automatically from the training data acts as an added advantage over manual methods. This capability of these models to convert the image into a set of features can be exploited to use them as feature extractors. In addition to the HC features, the extraction of features using DLM also is proposed.

For the purpose of extracting features using a DLM, the first step is to train the neural network. In this paper, EfficientNet-B0 and Xception are used as feature extractors. Each of these models are initialized with pre-trained ImageNet [19] weights. To train these deep learning models, the dataset is divided into 3 subsets - training (60\%), validation (20\%) and testing $(20 \%)$ set. After the model, a global max pooling layer is appended which finds the maximum value in the spatial dimension of the image at the last layer of the DLM. The output of the pooling layer is then flattened and fed into a dense layer of size same as the number of classes. The dense layer has SoftMax activation function due to multi-class classification problem.

Each network is trained on the input images rescaled to a size of $512 \times 512$ due to memory limitations. The images fed into the network are original WSIs without segmentation. This is because the DLM being an automatic feature extractor has the ability to itself segment and identify areas of interests. Thus, these networks can work on original images without the need for pre-processing of any form. In case of EfficientNet-B0, the output is of dimension $16 \times 16$ $\times 1280$ which is then subjected to global max pooling, converting the output to size 1280 . Thus, a total of 1280 features are obtained when EfficientNet-B0 is used as feature extractor as shown in Fig. 4. On the other hand, the output of Xception is of dimensions $32 \times 32 \times 2048$, which results in 2048 features after the application of global pooling as shown in Fig. 5.

\subsection{Feature selection}

The features identified through the two aforementioned methods are combined together for each image and two sets of feature vectors are obtained. The first set contains feature vectors 
5
5
㟔
5 交
○

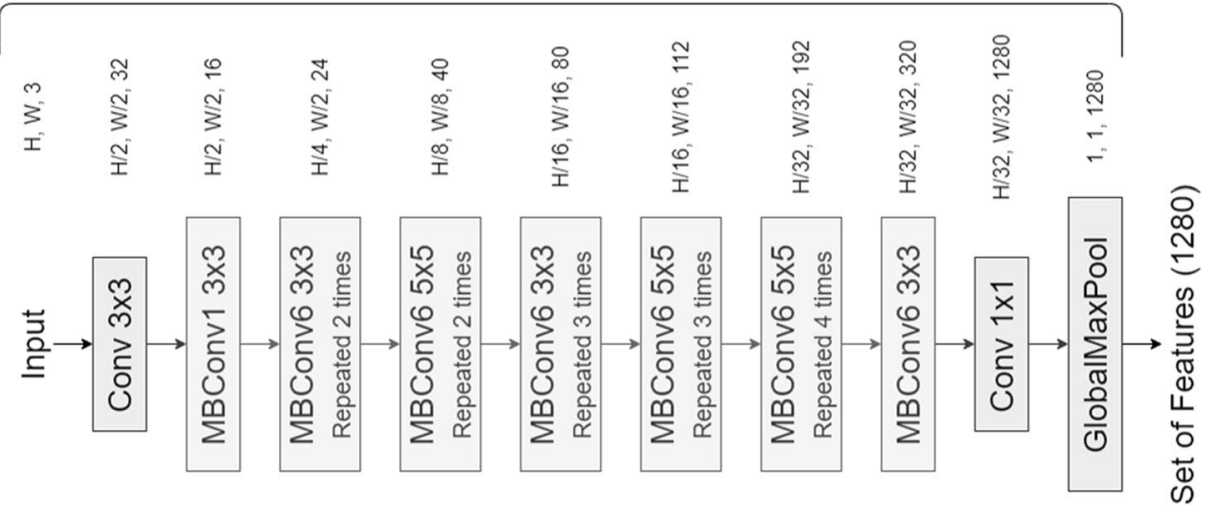

Fig. 4 Architecture of EfficientNet-B0 as feature extractor

obtained by the fusion of features extracted from $\mathrm{HC}$ methods and EfficientNet-B0. The size of feature vector obtained is 1350 . The second set contains feature vectors obtained by the fusion of features extracted from HC methods and Xception. The size of feature vector obtained is 2118. This results in huge pool of features for each image. But it is quite possible that not all of these features contribute to the final classification decision. So, it is important to select significant features from the pool of extracted features. Here, the proposed BAOA-S and BAOA-V explained in Section 5 to perform FS are employed.
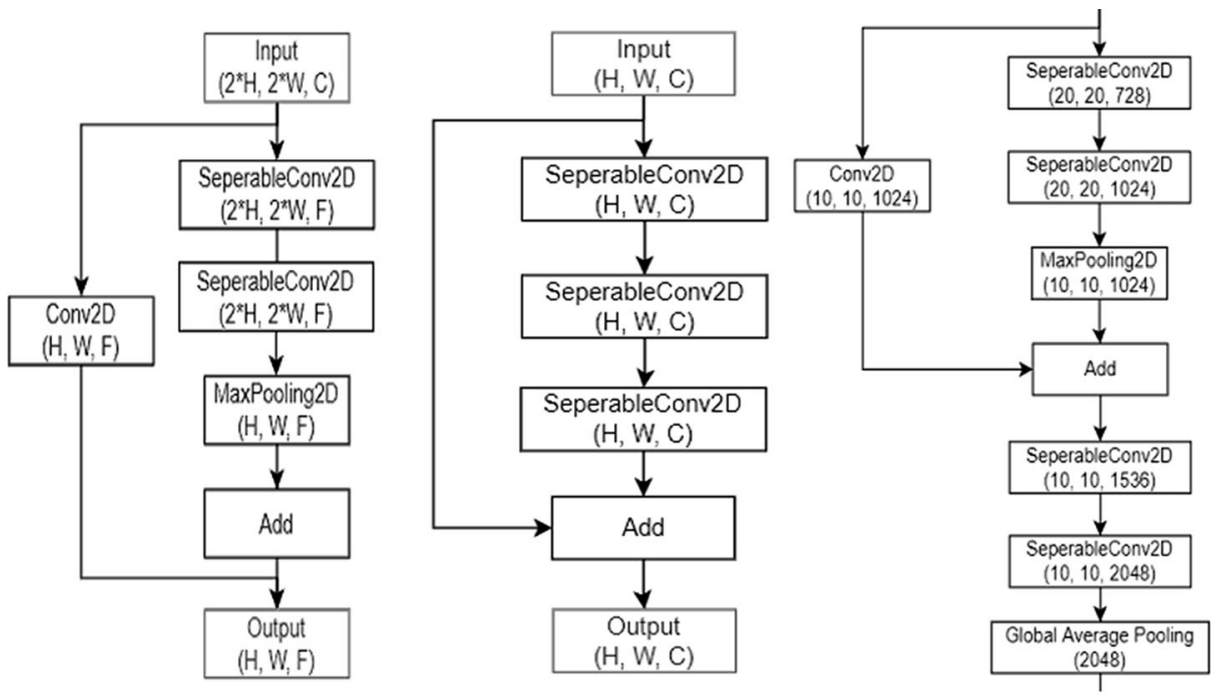

Fig. 5 Architecture of Xception as Feature Extractor (a) Initial Block to reduce image size by half, repeated 3 times, (b) Middle block, repeated 8 times, and (c) Final Layers 


\subsubsection{Generation of initial population}

BAOA-S/BAOA-V starts with the generation of initial population of binary feature vectors (FV) as shown in Fig. 6. Since the number of features chosen should also be uniformly distributed, a greedy method is employed to generate the set of initial solutions. The pseudo code of the greedy method used to generate initial population is shown in Fig. 7. A ' 1 ' in the feature vector shows that the corresponding feature is included during classification and a ' 0 ' shows that the corresponding feature is excluded.

\subsubsection{Fitness function}

In order to evaluate the quality of the feature vectors, the fitness of each FV is calculated. For this, the RBF-SVM classifier is trained with a set of features selected by that FV and then tested on the same set of features, but with the test set. Here, the objective is to maximize the classification accuracy and minimize the number of features. So, the fitness function is defined as shown in Eq. 15.

$$
\operatorname{Fitness}\left(F V_{i}\right)=\alpha E(C)+\beta \frac{|F|}{|N|}
$$

Where, Fitness $\left(F V_{i}\right)$ is the fitness of the $i^{\text {th }}$ solution vector, $E(C)$ is the classification error rate of RBF-SVM classifier, $|F|$ and $|N|$ are the number of features selected and the total number of features respectively, $\alpha \epsilon[0,1]$ and $\beta=(1-\alpha)$ are adopted from literature $[24,40]$.

The aim of applying BAOA-S and BAOA-V is to explore and exploit the solution space properly so as to obtain a set of features that minimizes the fitness function and increases the classification accuracy.

\subsection{Classification}

The final stage in the proposed approach is the classification of the WSIs into VT, NVT and NT. For this, once an optimal set of features are obtained, these are fed into a SVM classifier with RBF kernel. Once the training is completed, the test set is fed into the trained RBF-SVM classifier to detect osteosarcoma. The performance of RBF-SVM is measured using commonly applied performance metrics: specificity, sensitivity, precision, accuracy and ROC curve which are calculated using the values of True Positive (TP), True Negative (TN), False Positive (FN) and False Negative (FN).

Specificity is the ability to identify the healthy samples correctly and is calculated as:

$$
\text { Specificity }=\frac{T N}{T N+F P}
$$

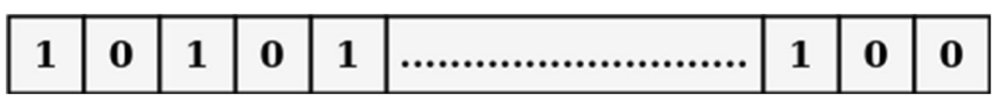

Fig. 6 Binary feature vector 
1. Repeat steps 1.1 to 1.3 for each feature vector $F V$.

1.1. Select a random integer $R \in U[1, N F]$, where, $N F$ is the total number of features extracted

1.2. Create a binary vector, with $R$ number of 1 's, rest 0 's, i.e.,

$$
F V=[1,1, \ldots(\mathrm{R} \text { times }), 0,0, \ldots(N F-\mathrm{R} \text { times })] .
$$

1.3. Shuffle the vector $F V$, to obtain a random sequence of 0 s and $1 \mathrm{~s}$.

2. The result will be a binary matrix, with each row representing a solution.

Fig. 7 Pseudo code of greedy method for population initialization

Sensitivity is the ability to determine the patients (TP) correctly and is calculated as:

$$
\text { Sensitivity }=\frac{T P}{T P+F N}
$$

Precision gives an estimate of how many samples are actually positive out of the total positive samples detected by the classifier

$$
\text { Precision }=\frac{T P}{T P+F P}
$$

Accuracy is the measure of number of images correctly classified (TP and TN) by the classifier and is calculated by:

$$
\text { Accuracy }=\frac{T P+T N}{T P+T N+F P+F N}
$$

ROC is a probability curve that graphically displays the true positives versus false positives across a range of thresholds settings. AUC is the area under the ROC curve and represents the

\begin{tabular}{|c|c|c|c|c|c|c|}
\hline \multirow[t]{2}{*}{ Class } & \multirow[t]{2}{*}{ Performance Metrics } & \multicolumn{5}{|c|}{ Feature Extraction Techniques } \\
\hline & & $\mathrm{HC}$ & EfficientNet-B0 & Xception & EfficientNet-B0 + HC & Xception + HC \\
\hline \multirow[t]{4}{*}{ VT } & Specificity & 0.945 & 0.9809 & 0.9814 & 0.9747 & 1. 0 \\
\hline & Sensitivity & 0.8636 & 0.9516 & 1. 0 & 0.9836 & 0.9839 \\
\hline & Precision & 0.9406 & 0.9516 & 0.9508 & 0.9375 & 1.0 \\
\hline & Accuracy & 0.9041 & 0.9726 & 0.9863 & 0.9772 & 0.9954 \\
\hline \multirow[t]{4}{*}{ NVT } & Specificity & 0.9515 & 0.9645 & 0.9643 & 0.9825 & 0.9826 \\
\hline & Sensitivity & 0.8889 & 0.96 & 0.9608 & 0.9583 & 0.9362 \\
\hline & Precision & 0.8571 & 0.8889 & 0.8909 & 0.9388 & 0.9362 \\
\hline & Accuracy & 0.9361 & 0.9635 & 0.9635 & 0.9772 & 0.9726 \\
\hline \multirow[t]{5}{*}{ NT } & Specificity & 0.939 & 0.9643 & 0.9817 & 0.9817 & 0.9633 \\
\hline & Sensitivity & 0.9455 & 0.9252 & 0.9182 & 0.9455 & 0.9727 \\
\hline & Precision & 0.8387 & 0.9612 & 0.9806 & 0.9811 & 0.964 \\
\hline & Accuracy & 0.9406 & 0.9452 & 0.9498 & 0.9635 & 0.968 \\
\hline & Overall Accuracy & 0.8904 & 0.9406 & 0.9497 & 0.9589 & 0.9608 \\
\hline
\end{tabular}
degree of separability. Higher the AUC higher is the degree of separability and vice-versa.

Table 1 Performance metrics of RBF-SVM for classification of WSIs into VT, NVT and NT using various combinations of feature extraction techniques

The values in bold show the best values obtained for various parameters. 


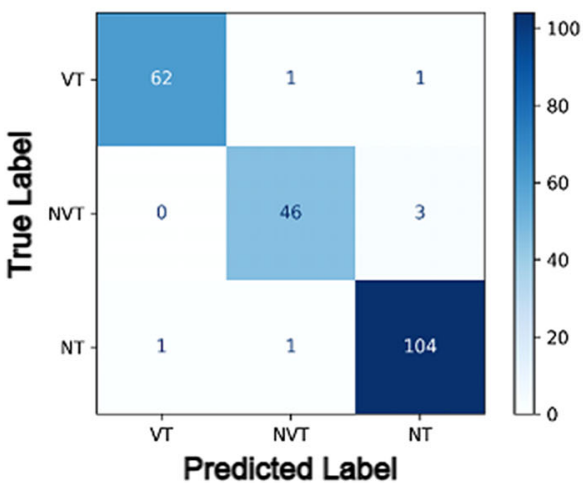

(a)

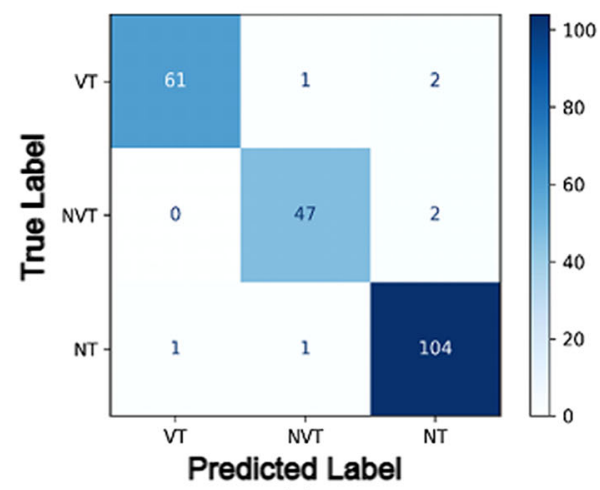

(c)

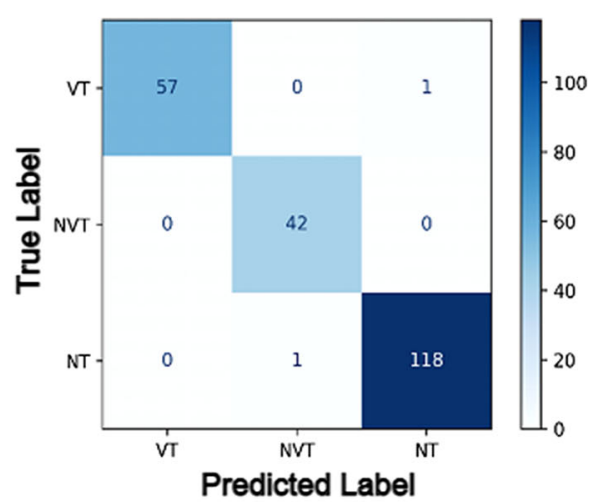

(b)

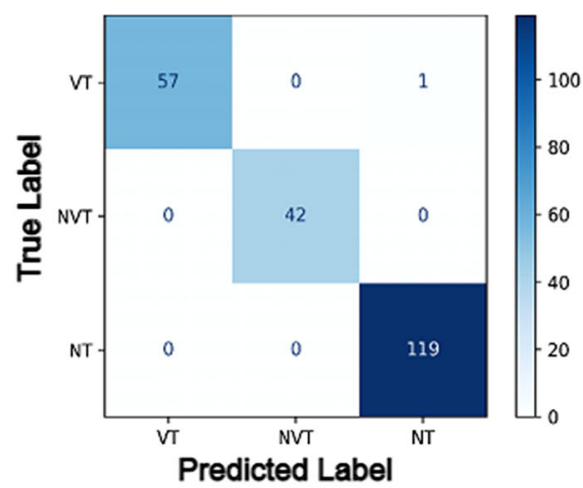

(d)

Fig. 8 Confusion matrix of classification of WSIs into VT, NVT and NT by RBF-SVM classifier using various combinations of feature extraction techniques and feature selection using BAOA-S (a) EfficientNet-B0, (b) Xception, (c) EfficientNet-B0 + HC, and (d) Xception $+\mathrm{HC}$

\section{Results and discussion}

In this section, we discuss the experimentation and analyse the results.

\subsection{Experimental setup and results analysis}

Here, a publicly available dataset that contains Hematoxylin and Eosin (H\&E) stained histological images of osteosarcoma [36] dataset comprises of 345 VT, 263 NVT and 536 NT images making a total of 1144 images is used. The images are of size $1024 \times 1024$.

Two sets of experiments are performed to compare the performance of various combinations of feature extraction techniques and to assess the impact of FS on RBF-SVM classifier's performance. The various combinations of features obtained by using different feature extraction techniques are (a) only HC features, (b) features extracted using EfficientNet-B0 only, (c) features extracted using Xception only, (d) combination of HC and EfficientNet-B0 features and (e) combination of HC and Xception features. The proposed model IF-FSM-C is implemented in Jupyter notebook in Python 3.7.10. Python libraries OpenCV, SciKit Learn and 


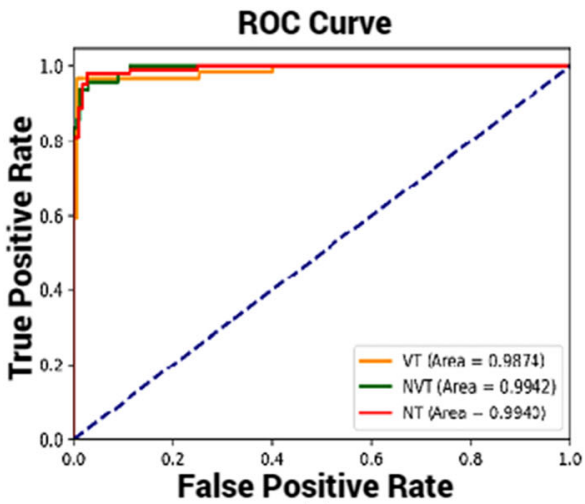

(a)

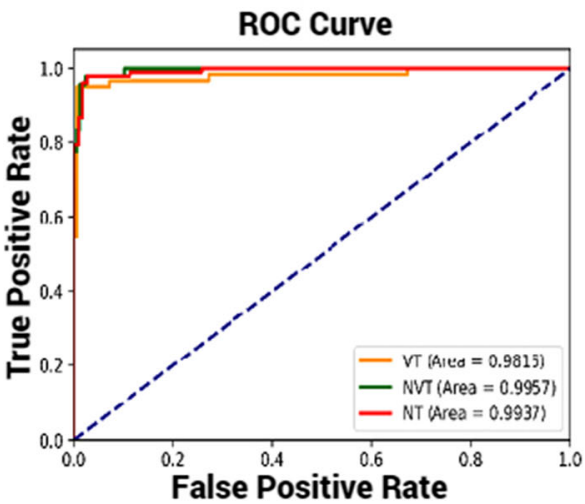

(c)

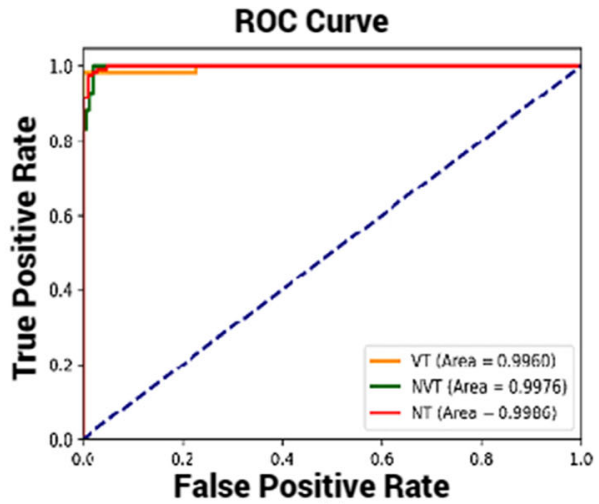

(b)

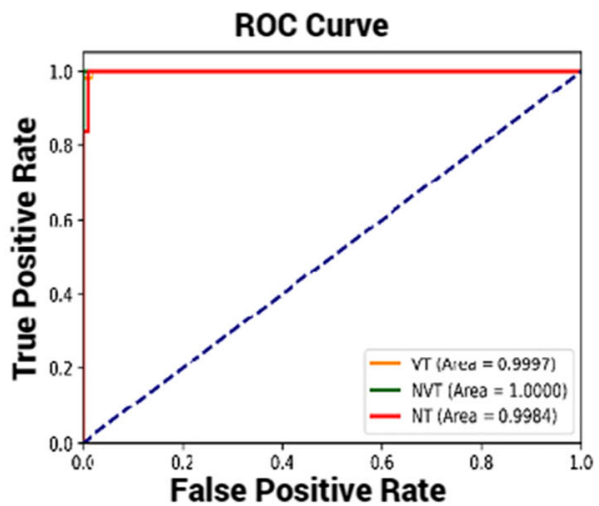

(d)

Fig. 9 ROC-AUC ofclassification of WSIs into VT, NVT and NT by RBF-SVM classifier using various combinations of feature extraction techniques and feature selection using BAOA-S (a) EfficientNet-B0, (b) Xception, (c) EfficientNet-B0 + HC, and (d) Xception $+\mathrm{HC}$

Numpy are used for image processing and Tensorflow with Keras framework for CNN training. The scripts are executed on Google Colab platform running on dual core Intel(R) Xeon(R) CPU @ 2.30GHz, and TPU acceleration for CNN training using Google Cloud TPUv2 (octa core). To fasten the process of training, the dataset is stored and retrieved using TFRecord which is a special format for storing a sequence of binary data. In our case, TFRecords contain sets of 32 images with their labels. TFRecords are created and stored on Google Cloud Storage (GCS) Bucket so that they can be easily fetched from the cloud thereby, reducing the processing time.

In the first set of experiments, the comparison of the performance of RBF-SVM when features are extracted using different combinations of feature extraction techniques and are given to the classifier without FS is done. The results are shown in Table 1.

When features extracted using DLMs are used for classification, the performance of the classifier is better as compared to when HC features are used alone as can be seen from Table 1. There is an improvement of 5.02\% and 5.93\% in the overall accuracy when EfficientNet-B0 and Xception are used respectively as feature extractor as compared to HC features. Also, the specificity, sensitivity and precision are very low when HC features are used as compared to DLM features. The classifier doesn't perform well on HC features because the 


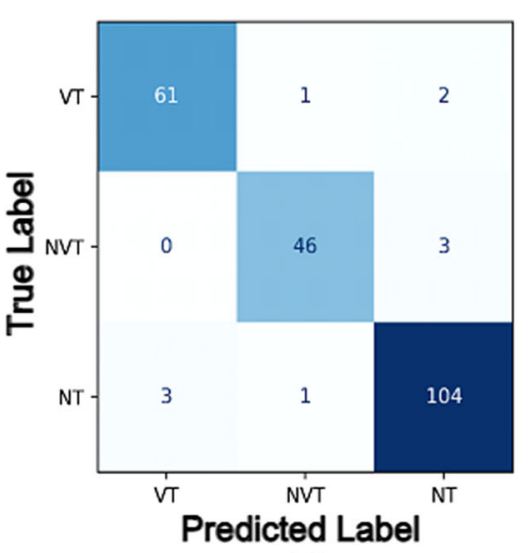

(a)

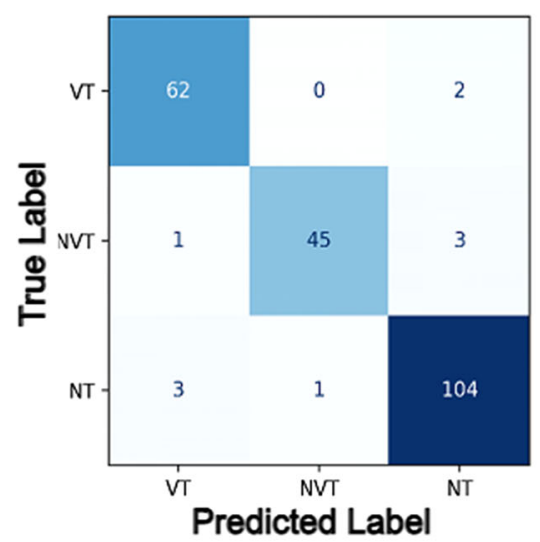

(c)

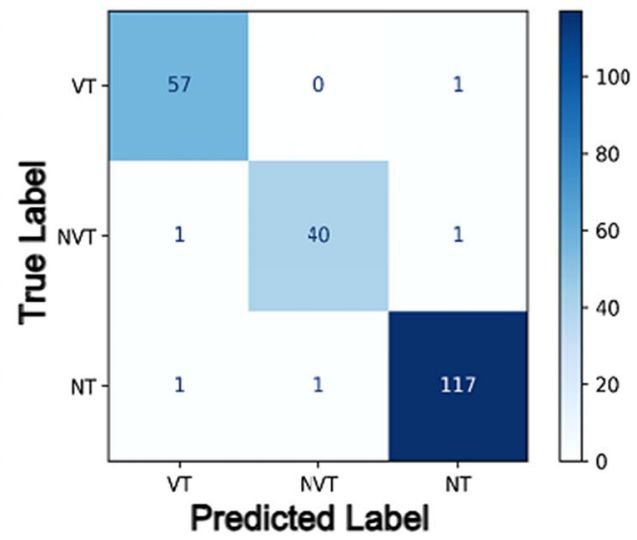

(b)

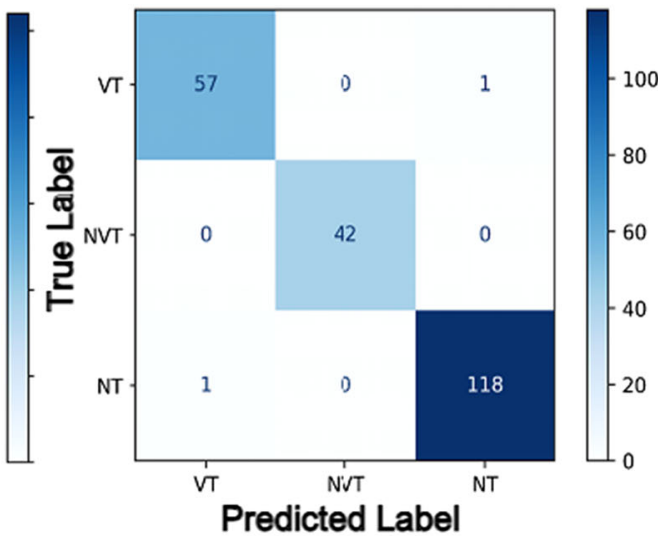

(d)

Fig. 10 Confusion matrix of classification of WSIs into VT, NVT and NT by RBF-SVM classifier using various combinations of feature extraction techniques and feature selection using BAOA-V (a) EfficientNet-B0, (b) Xception, (c) EfficientNet-B0 + HC, and (d) Xception $+\mathrm{HC}$

deep learning models, being automatic feature extractors, have the ability to identify low-level features which sometimes are skipped when HC features are used. This may lead to better performance on DLM features as compared to HC features only. When DLMs are compared, the features extracted using Xception leads to better classification of WSIs with an overall accuracy of $94.97 \%$ as compared to the accuracy of $94.06 \%$ achieved using EfficientNet-B0 features because Xception is a combination of ResNet and Inception networks, combining the strengths of both the networks, which leads to better identification of low-level features and consequently better results. On the other hand, EfficientNet majorly focuses on the efficient learning of the data and with this aim the accuracy may be compromised. Also, Xception as a feature extractor shows highest sensitivity in case of VT and NVT. However, when integrated $\mathrm{HC}$ and DLM features are used for classification, the classifier exhibits higher performance than when these features are used alone. It has been observed that without FS, the best performance is exhibited by integrated Xception and HC features with an overall accuracy of $96.08 \%$ which indicates that integrated features help in better classification of WSIs. The 


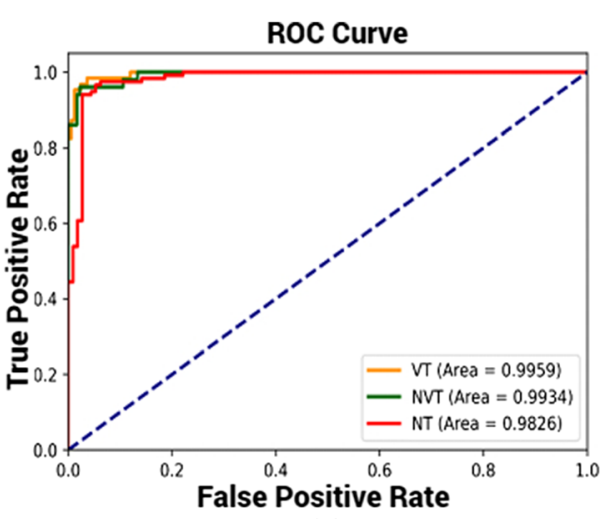

(a)

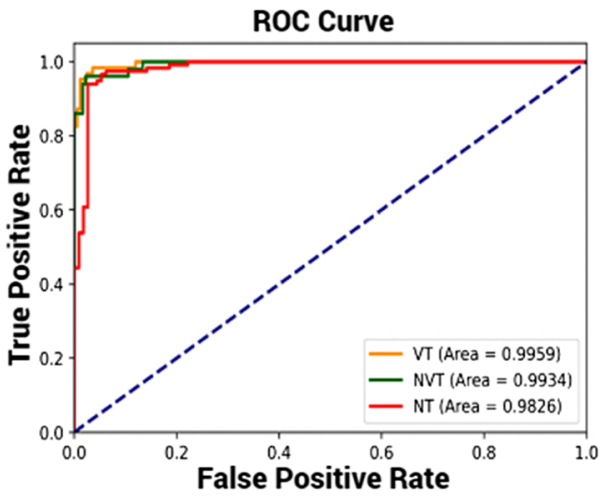

(c)

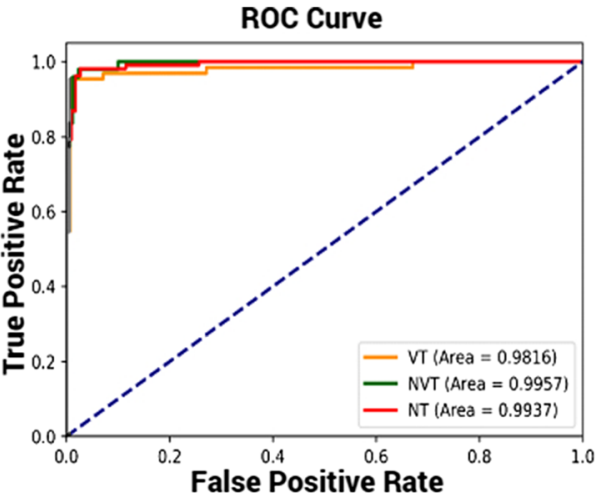

(b)

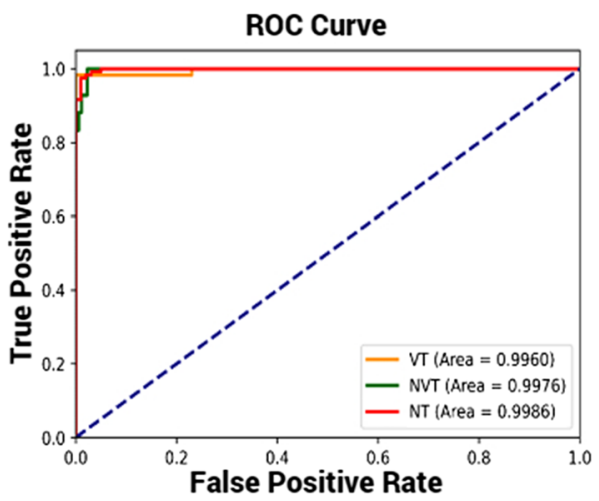

(d)

Fig. 11 ROC-AUC ofclassification of WSIs into VT, NVT and NT by RBF-SVM classifier using various combinations of feature extraction techniques and feature selection using BAOA-V (a) EfficientNet-B0, (b) Xception, (c) EfficientNet-B0 + HC, and (d) Xception + HC

high specificity, sensitivity and precision achieved using Xception and HC features exhibits the capability of the classifier in detecting healthy samples and patients correctly from the WSIs as compared to other techniques.

In the second set of experiments, FS is applied on the features extracted using different combinations of feature extraction techniques before giving it to RBF-SVM for classification. BAOA-S and BAOA-V are randomized algorithms, so these algorithms are run 30 times on each combination of features and report the best and average results obtained over 30 runs. The confusion matrix of RBF-SVM generated using the features obtained after applying BAOA-S for FS in the best case is shown in Fig. 8. The corresponding performance metrics and ROC-AUC curves are shown in Table 2; Fig. 9 respectively. The confusion matrix of RBF-SVM generated using the features obtained after applying BAOA-V in the best case is shown in Fig. 10. The corresponding performance metrics and ROC-AUC curves are shown in Table 3; Fig. 11 respectively. It is to be noted here that FS techniques are not applied in the case where HC features are used alone for classification. Finally, the average number of features selected over 30 


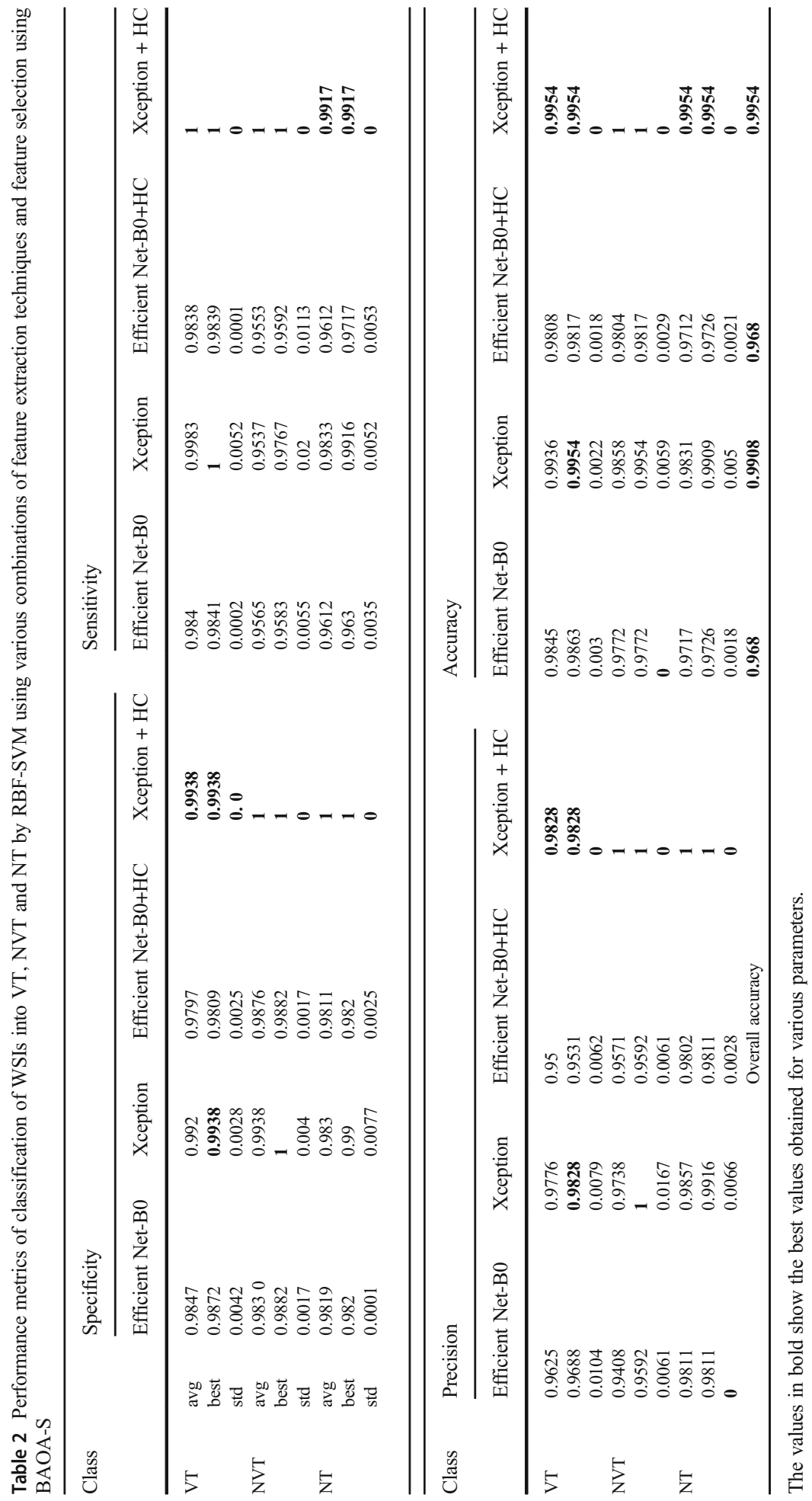




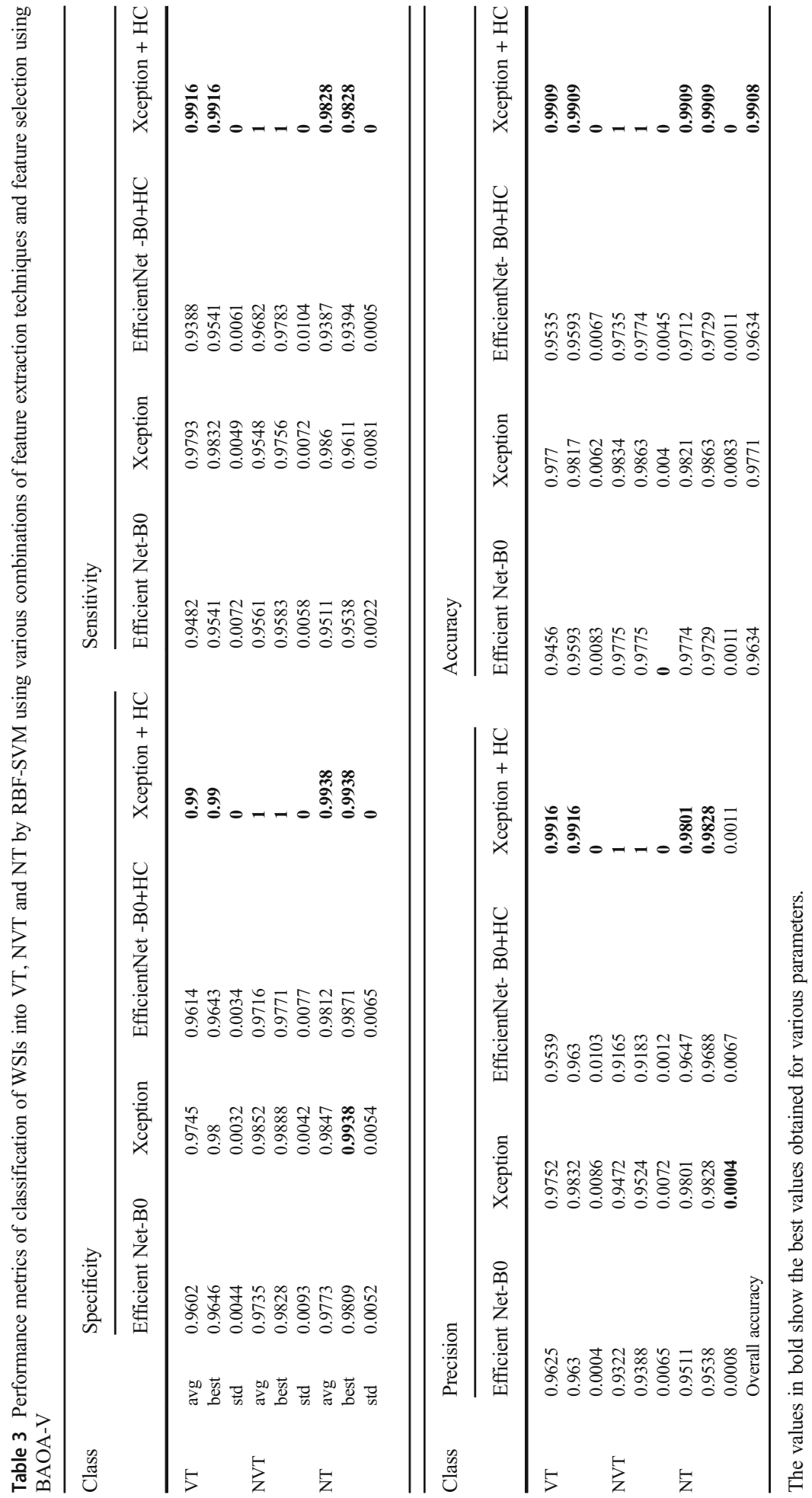


Table 4 Number of features selected using BAOA-S when features are extracted using a combination of different feature extraction techniques

\begin{tabular}{llllll}
\hline & & EfficientNet-B0 & Xception & EfficientNet-B0+HC & Xception + HC \\
\hline Number of Features & avg & 152.1 & 149.600 & 156.1 & 18.8 \\
& best & 799 & 1023 & 879 & 188 \\
& std & 304.6869 & 303.7618 & 315.2924 & 56.4 \\
\hline
\end{tabular}

runs of BAOA-S and BAOA-V and the number of features on which the best accuracy is obtained are shown in Tables 4 and 5 respectively.

When FS is applied on features extracted using HC techniques and DLM, it is observed from Tables 2 and 3 that it results in improvement in the performance of the classifier in case of both BAOA-S and BAOA-V. This is attributed to the fact that FS will keep only those features which are important and have a role in the classification process. The curse of redundant features may lead to decrease in the accuracy of a model and by the application of FS, the redundant or dependent features are removed, which leads to better results. It is also evident from Tables 2 and 3 that BAOA-S results in selection of better features as compared to BAOA-V. The best overall accuracy of $99.54 \%$ and above $99 \%$ specificity, sensitivity and precision, and highest AUC (VT-0.9997, NVT-1.00, NT-0.9984) is observed when integrated features (Xception+HC) with BAOA-S FS are used by the classifier as compared to other feature combinations. The performance of Xception with BAOA-S is comparable to that of integrated features (Xception+HC) with BAOA-S in case of VT images. However, integrated features (Xception+HC) with BAOA-S outperforms Xception with BAOA-S in case of NVT and NT. The number of average features selected by BAOA-S is less as compared to BAOA-V as evident from Tables 4 and 5, which indicates usage of lower computational resources and faster processing. These models can therefore, be applied in real time systems, where the results are expected in a fraction of time. The number of integrated Xception and HC features selected by applying BAOA-S on which the classifier shows the best performance is 188 as against 211 selected by applying BAOA-V.

Finally, two sets of Welch's t-test are performed to test whether the difference between the accuracy achieved by RBF-SVM is significant or not when the classifier uses (i) the integrated features (Xception + HC) selected using BAOA-S and BAOA-V and (ii) the features extracted using Xception with BAOA-S FS and integrated features (Xception+ HC) with BAOA-S FS, obtained over 30 runs. Here, Welch's t-test is performed on integrated features (Xception+HC) and Xception as their performance is comparable in case of VT images as compared to other feature extraction techniques used in this paper. Welch's t-test is performed at $\alpha=0.05$. The statistical result $(\mathrm{p}(2$-tailed $)=0.0216<0.05)$ on the first set shows that there is a significant difference between the performance of RBF-SVM when BAOA-S and BAOA-V FS are

Table 5 Number of features selected using BAOA-V when features are extracted using a combination of different feature extraction techniques

\begin{tabular}{llllll}
\hline & & EfficientNet-B0 & Xception & EfficientNet-B0+HC & Xception + HC \\
\hline Number of Features & avg & 238.5 & 341.3 & 265.2 & 47.8 \\
& best & 815 & 1543 & 912 & 211 \\
& std & 334.686 & 406.56 & 387.78 & 146.769 \\
\hline
\end{tabular}


Table 6 Comparison of class-wise and overall accuracy of state-of-the-art approaches with the proposed IFFSM-C model

\begin{tabular}{|c|c|c|c|c|c|c|c|}
\hline \multirow[t]{3}{*}{ Class } & & \multicolumn{6}{|c|}{ Accuracy in $\%$} \\
\hline & & \multirow[t]{2}{*}{$\begin{array}{l}\text { Mishra } \\
\text { et al.'s [42] } \\
\text { Deep } \\
\text { Learning } \\
\text { Model }\end{array}$} & \multicolumn{2}{|c|}{$\begin{array}{l}\text { Arunachalam } \\
\text { et al.’s [9] } \\
\text { Deep } \\
\text { Learning } \\
\text { Model }\end{array}$} & \multirow[t]{2}{*}{$\begin{array}{l}\text { Arunachalam et al.'s [9] } \\
\text { Expert- guided and } \\
\text { CellProfiler Features } \\
\text { (SVM) }\end{array}$} & \multirow[t]{2}{*}{$\begin{array}{l}\text { Anisuzzaman } \\
\text { et al.’s [7] Deep } \\
\text { Learning Model } \\
\text { (VGG19) }\end{array}$} & \multirow[t]{2}{*}{$\begin{array}{l}\text { IF-FSM-C } \\
\text { Model } \\
\text { (Xception } \\
+\mathrm{HC})\end{array}$} \\
\hline & & & Patch & Tile & & & \\
\hline \multirow[t]{4}{*}{ VT } & Specificity & 91 & - & - & 92 & 97.5 & 99.38 \\
\hline & Sensitivity & 92 & - & - & 92 & 92.85 & 100 \\
\hline & Precision & 92 & - & - & 93 & 94.20 & 98.28 \\
\hline & Accuracy & 92 & 95.3 & 92.6 & 91 & 96.09 & 99.54 \\
\hline \multirow[t]{4}{*}{ NVT } & Specificity & 89 & - & - & 0.86 & 97.19 & 100 \\
\hline & Sensitivity & 91 & - & - & 0.91 & 92.3 & 100 \\
\hline & Precision & 89 & - & - & 0.90 & 90.5 & 100 \\
\hline & Accuracy & 90 & 92.7 & 91.5 & 87 & 96.09 & 100 \\
\hline \multirow[t]{5}{*}{ NT } & Specificity & 93 & - & - & 0.93 & 95.90 & 100 \\
\hline & Sensitivity & 96 & - & - & 0.93 & 95.37 & 99.17 \\
\hline & Precision & 97 & - & - & 0.95 & 95.37 & 100 \\
\hline & Accuracy & 95 & 91.9 & 89.5 & 91 & 95.65 & 99.54 \\
\hline & $\begin{array}{l}\text { Overall } \\
\text { Accuracy }\end{array}$ & 92.4 & 93.3 & 91.2 & 89.9 & 93.91 & 99.54 \\
\hline
\end{tabular}

The values in bold show the best values obtained for various parameters.

applied on integrated features (Xception $+\mathrm{HC}$ ). The statistical result ( $\mathrm{p}$ (2-tailed) $=0.0364<0.05)$ on the second set shows that there is a significant difference between the performance of RBF-SVM when integrated features (Xception+HC) with BAOA-S are used as compared to when Xception features with BAOA-S FS are used alone.

\subsection{Comparison with State-of-the-Art Techniques}

The comparison of the proposed IF-FSM-C with the results reported in literature is shown in Table 6.

It is clear from Table 4 that the proposed IF-FSM-C outperforms the existing state-of-theart approaches in osteosarcoma detection. Arunachalam et al. [9] used expert-guided and CellProfiler features which are not sufficient enough to classify the WSIs as they don't extract low-level details. This leads to lower accuracy (89.9\%) of classification. Many researchers [7, 9 , 42] used DLMs for feature extraction and classification. Unlike HC feature extraction techniques, they result in the extraction of low-level features which can describe the image in greater detail but their capability is limited by the size of the dataset. The highest accuracy achieved using deep leaning models in the existing state-of-art- techniques is $93.91 \%$ [7]. In the work presented in this paper, more complex CNN models are used as feature extractor and a maximum overall accuracy of $94.97 \%$ is achieved with Xception as feature extractor without FS on RBF-SVM as classifier. This is further increased by $4.11-99.08 \%$ when FS is performed using BAOA-S. However, the highest overall accuracy of $99.54 \%$ is attained by the proposed IF-FSM-C (Xception + HC) model which is attributed to the fact that it uses integrated features 
thereby masking the limitation of deep learning models. The class accuracy, specificity, sensitivity and precision are highest in the case of the NVT class, which is marginally higher than VT and NT classes. This is due to the extremities of the two classes, and the NVT class being intermediate between VT and NT class, gets better trained than them. The proposed IFFSM-C model (Xception+ HC) also has higher specificity and sensitivity as compared to existing state of the art algorithms. In case of VT, the sensitivity is $100 \%$ which reflects the capabilities of the proposed model in detecting osteosarcoma patients correctly, without manual intervention, which is a very important feature of an automatic detection system.

The main limitation of the proposed model is the requirement of colored input images as the intensity of RGB channels are used during segmentation as well as during isolation of nuclei. The proposed method cannot be applied on MRI or CT scans as they do not contain microscopic information like nuclei density, nuclei shape etc. which are important in the detection of osteosarcoma. Another limitation is the requirement of large dataset for training the deep leaning models properly.

\section{Conclusion and future work}

In this paper, an automatic detection system Integrated Features-Feature Selection Model for Classification (IF-FSM-C) that detect osteosarcoma from the high-resolution whole slide images (WSIs) is proposed. Here, a fusion of features extracted using $\mathrm{HC}$ techniques and DLMs namely EfficientNet-B0 and Xception are used for classification by RBF-SVM. A FS step is applied before giving the integrated features to the classifier in order to remove redundant and irrelevant features. Two binary variant BAOA-S and BAOA-V of recently proposed AOA are proposed and applied for FS. The results indicates that integrated features along with FS by BAOA-S results in significant improvement in the performance of the classifier as compared to when $\mathrm{HC}(89.04 \%)$ are used alone or deep learning features are used alone with or without FS. In case of classifiers using integrated features, the performance of classifier on integrated Xception and HC features (99.54\%) with BAOA-S is better than that of integrated EfficientNet-B0 and HC features (96.8\%) with BAOA-S. When BAOA-S and BAOA-V are compared, it is observed that BAOA-S leads to better and a smaller number of features as compared to BAOA-V. As compared to existing state-of-the-art algorithms, IFFSM-C achieves $5.63 \%$ higher overall accuracy in osteosarcoma detection.

In future, we plan to explore other deep learning models which has a performance comparable to the current models and is computationally cheaper. This may help to further reduce the computational load and increase the speed of the system.

Data availability Data available on request from the authors.

Code availability Code available on request from the authors.

\section{Declarations}

Conflicts of interest/Competing interests There is no conflict of interest. 


\section{References}

1. Abdel-Basset M et al (2020) A new fusion of grey wolf optimizer algorithm with a two-phase mutation for feature selection. Expert Syst Appl 139:112824. https://doi.org/10.1016/j.eswa.2019.112824

2. Abualigah L, Diabat A, Mirjalili S, Elaziz MA, Gandomi AH (2021) The arithmetic optimization algorithm. Comput Methods Appl Mech Eng 376:113607

3. Adams R, Bischof L (1994) Seeded region growing. IEEE Trans Pattern Anal Mach Intell 16(6):641-647

4. Al-Tashi Q et al (2020) Binary multi-objective grey wolf optimizer for feature selection in classification. IEEE Access 8:106247-106263

5. Almaraz-Damian J-A, Ponomaryov V, Sadovnychiy S, Castillejos-Fernandez H (2020) Melanoma and nevus skin lesion classification using handcraft and deep learning feature fusion via mutual information measures. Entropy 22:484

6. Altameem T (2020) Fuzzy rank correlation-based segmentation method and deep neural network for bone cancer identification. Neural Comput Appl 32:805-815. https://doi.org/10.1007/s00521-018-04005-8

7. Anisuzzaman DM, Barzekar H, Tong L, Luo J, Yu Z (2020) A deep learning study on osteosarcoma detection from histological images. https://arxiv.org/pdf/2011.01177.pdf

8. Arora S, Anand P (2019) Binary butterfly optimization approaches for feature selection. Expert Syst Appl 116:147-160

9. Arunachalam HB, Mishra R, Daescu O et al (2019) Viable and necrotic tumor assessment from whole slide images of osteosarcoma using machine-learning and deep-learning models. PLoS One 14(4):e0210706. https://doi.org/10.1371/journal.pone.0210706

10. Arunachalam HB, Mishra R, Armaselu B, Daescu O, Martinez M, Leavey PJ, Rakheja D, Cederberg KB, Sengupta AL, Ni'suilleabhain M (2017) Computer aided image segmentation and classification for viable and non-viable tumor identification in osteosarcoma. Pacific Symposium on Biocomputing 195-206

11. Bakheet S (2017) An svm framework for malignant melanoma detection based on optimized hog features. Computation 5(1):4

12. Bansal P, Kumar S, Pasrija S, Kumar S (2020) A hybrid grasshopper and new cat swarm optimization algorithm for feature selection and optimization of multi-layer perceptron. Soft Comput 24:15463-15489

13. Bansal P, Kumar S, Srivastava R, Agarwal S (2021) Using transfer learning and hierarchical classifier to diagnose melanoma from dermoscopic images. Int J Healthc Inf Syst Inform (IJHISI) 16(2):73-86

14. Beucher S, Meyer F (1993) The morphological approach to segmentation: the watershed transformation. In Mathematical Morphology in Image Processing (Ed.E. R. Dougherty), 433-481

15. Bisla D, Choromanska A, Berman RS, Stein JA, Polsky D (2019) Towards automated melanoma detection with deep learning: Data purification and augmentation. In Proceedings - 2019 IEEE/CVF Conference on Computer Vision and Pattern Recognition Workshops, CVPRW 2019, 2720-2728

16. Cao Z, Duan L, Yang G (2019) An experimental study on breast lesion detection and classification from ultrasound images using deep learning architectures. BMC Med Imaging 19:51

17. Chen CX et al (2013) Osteosarcoma segmentation in MRI based on Zernike moment and SVM. Chinese J Biomed Eng 22(2):70-78

18. Chollet F (2016) Xception: Deep learning with depthwise separable convolutions. arXiv preprint arXiv: 1610.02357

19. Deng J, Dong W, Socher R, Li Li-Jia, Li K, Fei-Fei L (2009) ImageNet: A large-scale hierarchical image database. IEEE Conference on Computer Vision and Pattern Recognition 248-255. https://doi.org/10.1109/ CVPR.2009.5206848

20. Ding C, He X, Zha H, Simon HD (2002) Adaptive dimension reduction for clustering high dimensional data. Proceedings of International Conference on Data Mining

21. Elgamal ZM, Yasin NBM, Tubishat M, Alswaitti M, Mirjalili S (2020) An improved harris hawks optimization algorithm with simulated annealing for feature selection in the medical field. IEEE Access 8:186638-186652

22. El-Kenawy EM, Eid MM, Saber M, Ibrahim A (2020) MbGWO-SFS: Modified binary grey wolf optimizer based on stochastic fractal search for feature selection. IEEE Access 8:107635-107649

23. El-Kenawy E-SM, Ibrahim A, Mirjalili S, Eid MM, Hussein SE (2020) Novel feature selection and voting classifier algorithms for COVID-19 classification in CT images. IEEE Access 8:179317-179335

24. Emary E, Zawbaa HM, Hassanien AE (2016) Binary grey wolf optimization approaches for feature selection. Neurocomputing 172:371-381

25. Goode A, Gilbert B, Harkes J, Jukic D, Satyanarayanan M (2013) OpenSlide: A vendor-neutral software foundation for digital pathology. J Pathol Inform 4:27. https://doi.org/10.4103/2153-3539.119005

26. Hasan AM, Jalab HA, Meziane F, Kahtan H, Al-Ahmad AS (2019) Combining deep and handcrafted image features for MRI brain scan classification. IEEE Access 7:79959-79967 
27. He K, Zhang X, Ren S, Sun J (2016) Deep residual learning for image recognition. In Proceedings of CVPR, 770-778

28. Howard A, Zhu M, Chen B, Kalenichenko D, Wang W, Weyand T, Andreetto M, Adam H (2017) Mobilenets:Efficient convolutional neural networks for mobile vision applications. In CVPR

29. Hu P, Pan J-S, Chu S-C (2020) Improved Binary Grey Wolf Optimizer and Its application for feature selection. Knowl-Based Syst 195. https://doi.org/10.1016/j.knosys.2020.105746

30. Jia H, Zhao X, Qin L, Cai X (2021) Imaging method for osteosarcoma diagnosis and clinical staging information optimization. J Medi Imaging Health Infor 11(3):871-877

31. Kayal EB et al (2019) Segmentation of osteosarcoma tumor using diffusion weighted MRI: a comparative study using nine segmentation algorithms. Signal Image Video Process 14:727-735

32. Kennedy J, Eberhart R (1997) A discrete binary version of the particle swarm algorithm. In: Proceedings of the IEEE International Conference on Computational Cybernetics and Simulation

33. Khan MQ et al (2019) Classification of melanoma and nevus in digital images for diagnosis of skin cancer. IEEE Access 7:90132-90144

34. Kleinerman RA, Tucker MA, Tarone RE et al (2005) Risk of new cancers after radiotherapy in long-term survivors of retinoblastoma: an extended follow-up. J Clin Oncol 23:2272-2279

35. Krizhevsky A, Sutskever I, Hinton GE (2012) ImageNet classification with deep convolutional neural networks. Neural Information Processing Systems

36. Leavey P, Sengupta A, Rakheja D, Daescu O, Arunachalam HB, Mishra R (2019) Osteosarcoma data from UT Southwestern/UT Dallas for Viable and Necrotic Tumor Assessment [Data set]. The Cancer Imaging Archive

37. LeCun Y, Boser B, Denker JS, Henderson D, Howard RE, Hubbard W, Jackel LD (1989) Backpropagation applied to handwritten zip code recognition. Neural Comput 1(4):541-551

38. Li Y, Deng L, Yang X (2019) Early diagnosis of gastric cancer based on deep learning combined with the spectral-spatial classification method. Biomed Opt Express 10(10):4999-5014

39. Lindsey BA, Markel JE, Kleinerman ES (2017) Osteosarcoma overview. Rheumatol Ther 4(1):25-43

40. Mafarja M, Aljarah I, Faris H, Hammouri AI, Al-Zoubi Ala 'M, Mirjalili S (2018) Binary grasshopper optimisation algorithm approaches for feature selection problems. Expert Syst Appl 117:267-286

41. Mirabello L, Troisi RJ, Savage SA (2009) Osteosarcoma incidence and survival rates from 1973 to 2004 : data from the Surveillance, Epidemiology, and End Results Program. Cancer 115(7):1531-1543

42. Mishra R, Daescu O, Leavey P, Rakheja D, Sengupta A (2018) Convolutional neural network for histopathological analysis of osteosarcoma. J Comput Biol 25(3):313-325

43. Mishra R, Daescu O, Leavey P, Rakheja D, Sengupta A (2017) Histopathological diagnosis for viable and non-viable tumor prediction for osteosarcoma using convolutional neural network. International Symposium on Bioinformatics Research and Applications, Springer, Cham

44. Nasor M, Obaid W (2021) Segmentation of osteosarcoma in MRI images by K-means clustering, ChanVese segmentation, and iterative Gaussian filtering. IET Image Process

45. Ojala T, Pietikainen M, Maenpaa T (2002) Multiresolution gray-scale and rotation invariant texture classification with local binary patterns. IEEE Trans Pattern Anal Mach Intell 24(7):971-987

46. Otsu N (1979) A threshold selection method from gray-level histograms. IEEE Trans Syst Man Cybern 9(1):62-66

47. Qureshi MN, Ahamad MV (2018) An improved method for image segmentation using K-Means clustering with Neutrosophic logic. Procedia Comput Sci 132:534-540

48. Rahmawaty M, Nugroho HA, Triyani Y, Ardiyanto I, Soesanti I (2016) Classification of breast ultrasound images based on texture analysis. International Conference on Biomedical Engineering (iBioMed), 1-6

49. Rashedi E, Nezamabadi-pour H, Saryazdi S (2009) BGSA: binary gravitational search algorithm. Nat Comput 9(3):727-745

50. Samet H (2006) Foundations of multidimensional and metric data structures. Morgan Kaufmann, Burlington

51. Seigel R et al (2021) Cancer Statistics 2021. Cancer J Clin 71(1):7-33

52. Shankar K, Perumal E (2020) A novel hand-crafted with deep-learning features based fusion model for COVID-19 diagnosis and classification using chest X-ray images. Complex Intell Syst 7:1277-1293. https://doi.org/10.1007/s40747-020-00216-6

53. Solmaz A, Tajeripour F (2016) Detection of brain tumor in 3D MRI images using local binary patterns and histogram orientation gradient. Neurocomputing 219

54. Srinivasu PN, SivaSai JG, Ijaz MF, Bhoi AK, Kim W, Kang JJ (2021) Classification of skin disease using deep learning neural networks with MobileNet V2 and LSTM. Sensors 21(8):2852

55. Szegedy C, Liu W, Jia Y et al (2015) Going deeper with convolutions. In IEEE Conference on Computer Vision and Pattern Recognition (CVPR) 
56. Tan M, Le QV (2019) EfficientNet: Rethinking model scaling for convolutional neural networks. In Proceedings of International Conference on Machine Learning (ICML)

57. Thaher T, Heidari AA, Mafarja M, Dong JS, Mirjalili S (2020) Binary Harris Hawks optimizer for highdimensional, low sample size feature selection. In: Mirjalili S, Faris H, Aljarah I (eds) Evolutionary Machine Learning Techniques. Algorithms for Intelligent Systems. Springer, Singapore. https://doi.org/10.1007/978981-32-9990-0 12

58. Wang H, Oliensis $\mathrm{J}$ (2010) Generalizing edge detection to contour detection for image segmentation. Comput Vis Image Underst 114:731-744

59. Wolpert DH, Macready WG (1997) No free lunch theorems for optimization. IEEE Trans Evol Comput 1(1):67-82

60. Yadav D, Kohli N, Agarwal A, Vatsa M, Singh R, Noore A (2018) Fusion of handcrafted and deep learning features for large-scale multiple iris presentation attack detection. In IEEE Conference on Computer Vision and Pattern Recognition Workshops

61. Zhang R et al (2018) Multiple supervised residual network for osteosarcoma segmentation in CT images. Comput Med Imaging Graphics 63:1-8

Publisher's Note Springer Nature remains neutral with regard to jurisdictional claims in published maps and institutional affiliations.

\section{Affiliations}

\section{Priti Bansal $^{1} \cdot$ Kshitiz Gehlot $^{2} \cdot$ Abhishek Singhal $^{2} \cdot$ Abhishek Gupta $^{3}$}

Abhishek Gupta

abhishekgupta10@yahoo.co.in

Priti Bansal

bansalpriti79@gmail.com

Kshitiz Gehlot

kshitizg99@gmail.com

Abhishek Singhal

abhisheksinghal.18199@gmail.com

1 Department of Information Technology, Netaji Subhas University of Technology, Dwarka, New Delhi, India

2 Department of Information Technology, Netaji Subhas Institute of Technology, New DelhiDwarka, India

3 School of Computer Science and Engineering, Shri Mata Vaishno Devi University, Katra, Jammu and Kashmir, India 